\section{VARGEN: A multiple regression and correlation teaching program}

\author{
ROBERT G. St. PIERRE \\ Boston College, Newton, Massachusetts 02167
}

VARGEN (variable generator) creates sets of data with known statistical properties by generating user-specified variables which are functions of uniformly distributed random numbers for each of a group of Ss. The user specifies the relative size and location of from one to nine predictor variables and one criterion variable within a 10 by 10 matrix, and, therefore, the amount of variance accounted for by each variable. For each S, 100 random numbers are drawn from a rectantular distribution which ranges from zero to one. The random numbers are placed in the cells of a 10 by 10 matrix with the numbers contained in the cells that are covered by each variable being summed. A variable is thus defined as the sum of equally potent, equally likely, independent elements, which are either present or absent (McNemar, 1969; Garrett, 1946). A visual display is produced showing the size and location of any five variables.

While a multiple correlation and regression routine (Cooley \& Lohnes, 1971) has been built into VARGEN to enable the student to explore relationships between the generated variables, the program has the option to punch the generated data on cards permitting its input to any data analysis program. Since the user has specified the relationships between variables, many statistical characteristics of the data are known, and certain results can be expected from an analysis, enabling the data to be used as part of a learning process.

Effects of varying sample size, adding or deleting variables and varying the relationships between variables can be observed. The main purpose of VARGEN is as an educational tool to enable students to deepen their understanding of the statistical properties of data.

Input. VARGEN requires two input cards for each pass through the program. One card describes the number of $\mathrm{Ss}$, variables to be visually displayed and other options. The second card describes the relative size and location of each variable within the 10 by 10 matrix.

Output. (1) Number of variables and number of Ss. (2) Size and location of each variable. (3) Visual display of any five variables. Gives a representation of the 10 by 10 matrix and the area covered by each variable. (4) Listing of generated data (optional). (5) Output of generated data to Logical Unit 7 (optional). (6) Mean and standard deviation for each variable. (7) Correlation matrix. (8) Predictor inverse. (9) Multiple regression analysis. (10) Formulas used in regression analysis.

Computer and Language. VARGEN is written in IBM FORTRAN IV G and requires the subroutine RANDU from the IBM Scientific Subroutine Package. It was developed on an IBM 370/45, and has been successfully adapted to a Systems Engineering Laboratory 7200 . VARGEN can be adapted to other computers which have FORTRANIVG compilers, but the random number generating subroutine must be specific to the word length of the particular computer.

Availability. A users manual, program listing, sample output and source deck may be obtained free from Robert G. St. Pierre, 224 Newtonville Avenue, Newton, Massachusetts 02158.

\section{REFERENCES}

Cooley, W., \& Lohnes, P. Multivariate data analysis. New York: Wiley, 1971

Garrett, H. Statistics in psychology and education. New York: Longmans, Green, 1946.

McNemar, Q. Psychological statistics. New York: Wiley, 1969. 